\title{
A case of pituitary abscess presenting without a source of infection or prior pituitary pathology
}

\author{
Derick Adams and Philip A Kern
}

Division of Endocrinology and Molecular Medicine, University of Kentucky Medical Center, Lexington, Kentucky, USA

Correspondence should be addressed to D Adams

Email

derick.adams@uky.edu

\section{Summary}

Pituitary abscess is a relatively uncommon cause of pituitary hormone deficiencies and/or a suprasellar mass. Risk factors for pituitary abscess include prior surgery, irradiation and/or pathology of the suprasellar region as well as underlying infections. We present the case of a 22-year-old female presenting with a spontaneous pituitary abscess in the absence of risk factors described previously. Her initial presentation included headache, bitemporal hemianopia, polyuria, polydipsia and amenorrhoea. Magnetic resonance imaging (MRI) of her pituitary showed a suprasellar mass. As the patient did not have any risk factors for pituitary abscess or symptoms of infection, the diagnosis was not suspected preoperatively. She underwent transsphenoidal resection and purulent material was seen intraoperatively. Culture of the surgical specimen showed two species of alpha hemolytic Streptococcus, Staphylococcus capitis and Prevotella melaninogenica. Urine and blood cultures, dental radiographs and transthoracic echocardiogram failed to show any source of infection that could have caused the pituitary abscess. The patient was treated with 6 weeks of oral metronidazole and intravenous vancomycin. After 6 weeks of transsphenoidal resection and just after completion of antibiotic therapy, her headache and bitemporal hemianopsia resolved. However, nocturia and polydipsia from central diabetes insipidus and amenorrhoea from hypogonadotrophic hypogonadism persisted.

\section{Learning points:}

- Pituitary abscesses typically develop in patients who have other sources of infection or disruption of the normal suprasellar anatomy by either surgery, irradiation or pre-existing pathology; however, they can develop in the absence of known risk factors.

- Patients with pituitary abscesses typically complain of headache, visual changes and symptoms of pituitary hormone deficiencies.

- As other pituitary neoplasms present with similar clinical findings, the diagnosis of pituitary abscess is often not suspected until transsphenoidal resection is performed.

- Prompt surgical and medical treatment of pituitary abscess is necessary, which typically results in improvement in headache and visual changes; however, pituitary hormone deficiencies are typically often permanent.

\section{Background}

The patient described in this case had no source of infection, prior surgeries or other pathology of the pituitary. As this patient had no risk factors for pituitary abscess, the diagnosis was not suspected until the time of surgery. Preoperative diagnosis of pituitary abscess requires a high degree of clinical suspicion. This case will 
help make clinicians aware of this rare condition that has a similar presentation of other more common pathology of the pituitary.

\section{Case presentation}

A 22-year-old Caucasian female presented with headaches, visual changes, amenorrhoea, polydipsia, increased frequency of urination and nocturia for 4 months. She had no significant medical or dental history and was on no medications or nutritional supplements. No history of head trauma was reported. She admitted to increased thirst and stated that she consumed approximately $7-12 \mathrm{~L}$ of water per day for several months. She also reported nocturia and increased frequency of urination, but she was unable to quantify her urine output. She denied fever, chills or any symptoms of infection. She had no history of illicit or intravenous drug use. Her height was $175.3 \mathrm{~cm}$, weight was $77.1 \mathrm{~kg}$, heart rate was 86 and blood pressure was $120 / 82 \mathrm{mmHg}$. Her physical examination was unremarkable.

\section{Investigation}

Urine pregnancy test was performed and was negative. MRI revealed a suprasellar, peripherally enhancing, cystic mass measuring $19 \times 15 \times 22 \mathrm{~mm}$ with superior displacement of the optic chiasm (Fig. 1). The posterior pituitary bright spot was also absent on her MRI. The differential diagnosis of her suprasellar mass included pituitary macroadenoma, other suprasellar neoplasms such as craniopharyngioma, and pituitary abscess. Her initial biochemical testing is shown in Table 1. Formal visual field testing showed bitemporal hemianopsia. Her serum sodium remained in the normal range preoperatively. Water deprivation testing to confirm the diagnosis of central diabetes insipidus was not performed. As the patient described two to three episodes of nocturia per night, consumed 7-12 L of water per day and her MRI showed absence of the posterior pituitary bright spot, the diagnosis of central diabetes insipidus was highly probable.

\section{Treatment}

Signs or symptoms of adrenal insufficiency or hypothyroidism were not present, so glucocorticoids and levothyroxine were not initiated. Due to her compressive symptoms and pituitary hormone deficiencies, transsphenoidal resection was recommended

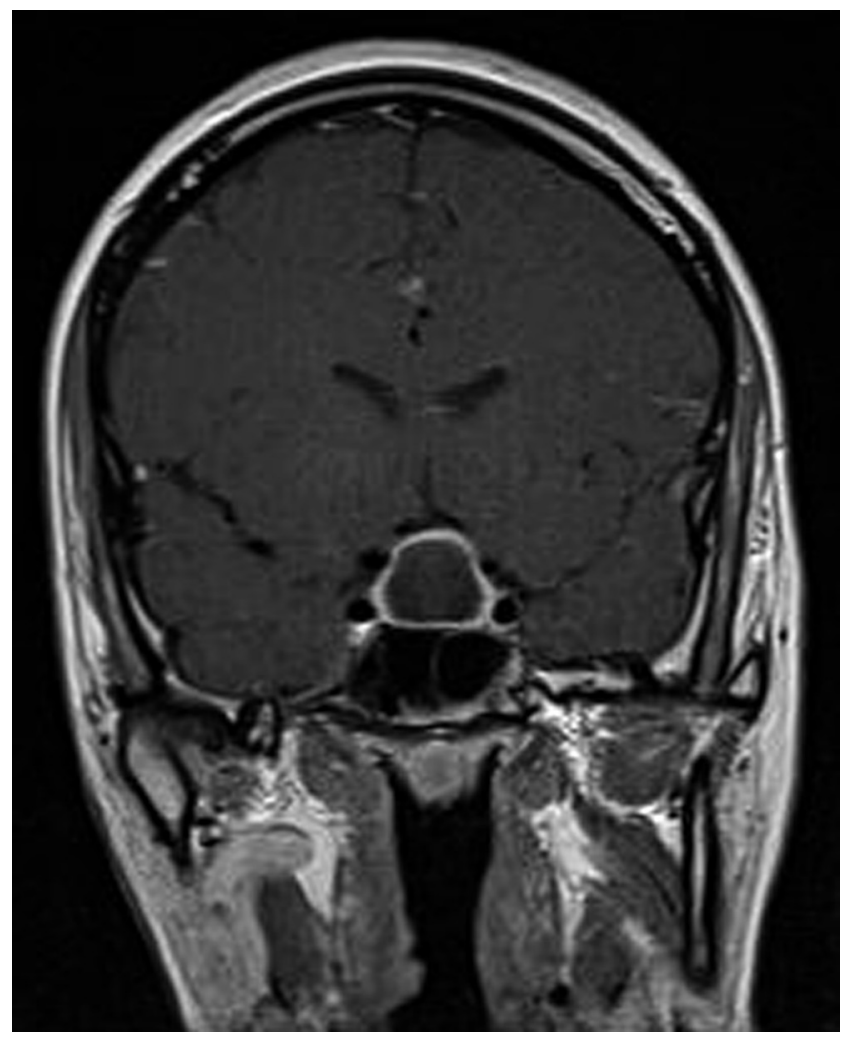

Figure 1

Preoperative T1-weighted coronal, magnetic resonance imaging after intravenous gadolinium contrast administration showing a suprasellar peripherally enhancing, cystic mass measuring $19 \times 15 \times 22 \mathrm{~mm}$ with superior displacement of the optic chiasm.

to the patient and she agreed to proceed with surgery. Transsphenoidal resection was performed 2 weeks after her initial presentation. The diagnosis of pituitary abscess was made when purulent material was encountered upon surgical incision of the dura mater that forms the capsule of the pituitary gland. Surgical pathology revealed benign anterior pituitary tissue with marked lymphoplasmacytic

Table 1 Patient's preoperative biochemistry results at $1500 \mathrm{~h}$.

\section{Test}

White blood cell count

Serum sodium

Urine-specific gravity

ACTH

Serum cortisol

TSH

Free $\mathrm{T}_{4}$

Follicle-stimulating

hormone

Luteinizing hormone

Prolactin

IGF-1

\begin{tabular}{c}
\hline Result \\
\hline $5.8 \mathrm{k} / \mu \mathrm{L}$ \\
$143 \mathrm{mmol} / \mathrm{L}$ \\
1.005 \\
$9 \mathrm{pg} / \mathrm{mL}$ \\
$2.7 \mu \mathrm{g} / \mathrm{dL}$ \\
$0.06 \mu \mathrm{IU} / \mathrm{mL}$ \\
$0.7 \mathrm{ng} / \mathrm{dL}$ \\
$4.1 \mathrm{mIU} / \mathrm{mL}$ \\
\\
$0.3 \mathrm{mIU} / \mathrm{mL}$ \\
$22.4 \mathrm{ng} / \mathrm{mL}$ \\
$301 \mathrm{ng} / \mathrm{mL}$ \\
\hline
\end{tabular}

\begin{tabular}{c}
\hline Normal range \\
\hline $3.7-10.3 \mathrm{k} / \mu \mathrm{L}$ \\
$136-145 \mathrm{mmol} / \mathrm{L}$ \\
$1.001-1.030$ \\
$6-50 \mathrm{pg} / \mathrm{mL}$ \\
$3-14 \mu \mathrm{g} / \mathrm{dL}$ \\
$0.4-4.2 \mu \mathrm{IU} / \mathrm{mL}$ \\
$0.8-1.7 \mathrm{ng} / \mathrm{dL}$ \\
$1.7-21.5 \mathrm{mlU} / \mathrm{mL}$ \\
\\
$1.0-95.6 \mathrm{mIU} / \mathrm{mL}$ \\
$4.4-23.3 \mathrm{ng} / \mathrm{mL}$ \\
$83-456 \mathrm{ng} / \mathrm{mL}$ \\
\hline
\end{tabular}


Table 2 Patient's biochemistry results 6 weeks postoperatively at $900 \mathrm{~h}$.

\begin{tabular}{|c|c|c|}
\hline Test & Result & Normal range \\
\hline Serum sodium & $147 \mathrm{mmol} / \mathrm{L}$ & $136-145 \mathrm{mmol} / \mathrm{L}$ \\
\hline Urine osmolality & $117 \mathrm{mOsm} / \mathrm{kg}$ & $300-900 \mathrm{mOsm} / \mathrm{kg}$ \\
\hline Serum cortisol & $8.1 \mu \mathrm{g} / \mathrm{dL}$ & $3-14 \mu \mathrm{g} / \mathrm{dL}$ \\
\hline $\begin{array}{l}\text { Serum cortisol } 1 \mathrm{~h} \text { after } \\
250 \mu \mathrm{g} \text { intramuscular } \\
\text { ACTH }\end{array}$ & $29.1 \mu \mathrm{g} / \mathrm{dL}$ & $>18.0 \mu \mathrm{g} / \mathrm{dL}$ \\
\hline TSH & $2.13 \mu \mathrm{IU} / \mathrm{mL}$ & $0.4-4.2 \mu \mathrm{IU} / \mathrm{mL}$ \\
\hline Free $\mathrm{T}_{4}$ & $0.9 \mathrm{ng} / \mathrm{dL}$ & $0.8-1.7 \mathrm{ng} / \mathrm{dL}$ \\
\hline $\begin{array}{l}\text { Follicle-stimulating } \\
\text { hormone }\end{array}$ & $6.1 \mathrm{mIU} / \mathrm{mL}$ & $1.7-21.5 \mathrm{mlU} / \mathrm{mL}$ \\
\hline Luteinizing hormone & $1.7 \mathrm{mlU} / \mathrm{mL}$ & $1.0-95.6 \mathrm{mIU} / \mathrm{mL}$ \\
\hline Estradiol & $30 \mathrm{pg} / \mathrm{mL}$ & $0-400 \mathrm{pg} / \mathrm{mL}$ \\
\hline IGF-1 & $169 \mathrm{ng} / \mathrm{mL}$ & $83-456 \mathrm{ng} / \mathrm{mL}$ \\
\hline
\end{tabular}

inflammation and fibrosis. Culture of the surgical specimen showed two species of alpha hemolytic Streptococcus, Staphylococcus capitis and Prevotella melaninogenica. After the diagnosis of pituitary abscess and before antibiotics were initiated, blood and urine cultures were collected but did not reveal any source of infection. Transthoracic echocardiogram was also performed, which did not reveal infectious endocarditis. Dental examination and dental radiographs were also performed; however, there was no evidence of a dental infection. Further review of the patient's MRI showed no radiographic signs of paranasal sinusitis. The patient was treated with 6 weeks of oral metronidazole and intravenous vancomycin based on her surgical cultures and sensitivities.

\section{Outcome and follow-up}

Postoperatively, her urine output increased to $440 \mathrm{~mL} / \mathrm{h}$ with serum sodium levels reaching $154 \mathrm{mmol} / \mathrm{L}$. She required administration of intravenous fluid and several doses of intravenous desmopressin to correct her polyuria and normalise her serum sodium levels. She was discharged from the hospital 5 days postoperatively with prescriptions for oral desmopressin at night, oral metronidazole $500 \mathrm{mg}$ every $8 \mathrm{~h}$, and intravenous vancomycin $1000 \mathrm{mg}$ every $8 \mathrm{~h}$ administered via a peripheral inserted central catheter. She was not discharged on corticosteroids or levothyroxine but instructed to call the on-call endocrine physician if she experienced symptoms of adrenal insufficiency or hypothyroidism. After 6 weeks of transsphenoidal resection and just after completion of antibiotic therapy, her headaches and bitemporal hemianopsia resolved; however, her diabetes insipidus and amenorrhoea

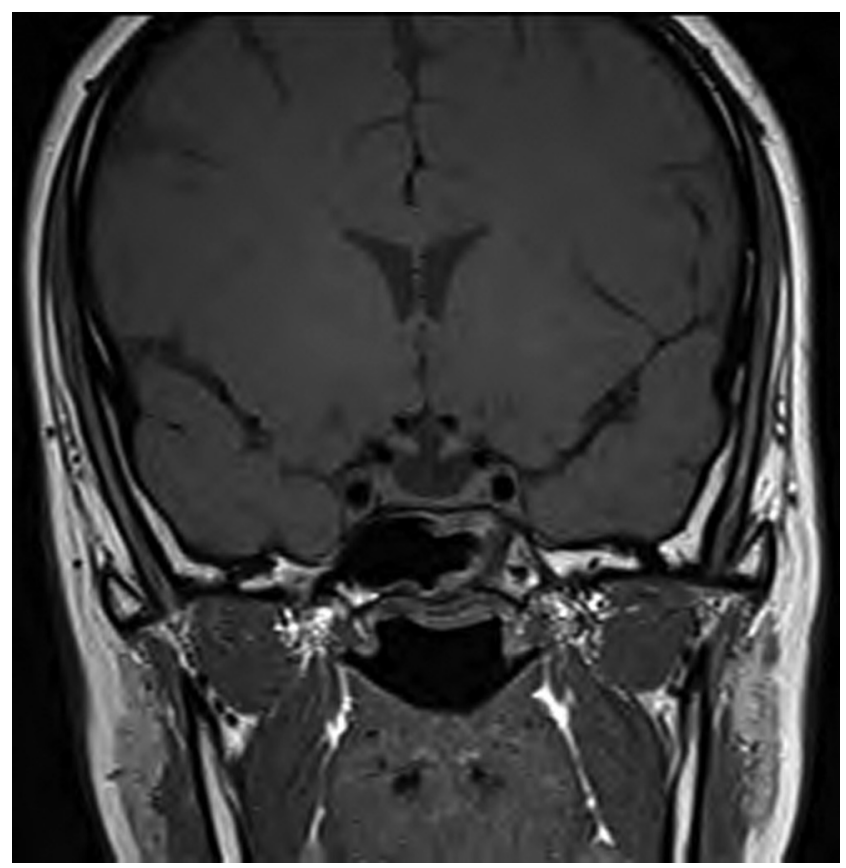

Figure 2

A 13-week, postoperative, T1-weighted coronal, magnetic resonance imaging after intravenous gadolinium contrast administration showing resolution of the pituitary abscess and postsurgical changes.

persisted. Her biochemical testing results 6 weeks after transsphenoidal resection are presented in Table 2 . Oestrogen and progesterone replacement was discussed with the patient. At 6 weeks after surgery, it was decided to delay oestrogen and progesterone replacement to see if she would have spontaneous resumption of menses. She was informed that if her menstrual cycles do not return, oestrogen and progesterone therapy would need to be initiated to prevent adverse effects such as bone loss. MRI performed 13 weeks postoperatively showed resolution of the pituitary abscess, postsurgical changes and absence of the posterior pituitary bright spot (Fig. 2). She still reported amenorrhoea, nocturia and polydipsia, requiring intranasal desmopressin at 32 weeks postoperatively.

\section{Discussion}

Pituitary abscesses are uncommon. Pituitary abscesses typically occur following disruption of the normal anatomy of the suprasellar region either by surgery, irradiation or a pathological process such as a neoplasm. The patient in this study did not have any history of pituitary surgery, irradiation or other pathological anatomic abnormalities. One case series of pituitary abscesses reported that $41.7 \%$ of patients with a pituitary 
abscess had undergone previous pituitary or sphenoid sinus surgery (1). Pituitary adenoma, Rathke's cleft cyst, craniopharyngioma and CSF fistula have been associated with pituitary abscess formation (1). It has been theorised that anatomical abnormalities of the pituitary may predispose patients to abscess formation due to impaired circulation, tissue necrosis or local immunological impairment (2).

Besides anatomic abnormalities, local or systemic infections are also known risk factors for pituitary abscess. As demonstrated in this case, patients with pituitary abscesses typically do not have an identifiable underlying infection. In one case series, only $16.7 \%$ of patients with a pituitary abscess had possible sepsis or sources of bacteraemia (1). The patient in this case underwent extensive evaluation for an underlying infection; however, no infection was identified. The patient did not receive antibiotics preoperatively, so this would not have resulted in false-negative blood and urine cultures.

Our patient sought medical care because of compressive symptoms and symptoms of pituitary hormone deficiencies. Headache is the most common symptom of pituitary abscess. In one series of 24 patients, 22 reported a headache (1). Visual changes, such as hemianopsia, are also relatively common. In one series, $68 \%$ of patients with pituitary abscess presented with visual changes (3). Pituitary hormone deficiencies are common as well. Thirty to fifty percent of patients with pituitary abscess have pituitary hormone deficiencies (4). The patient we presented had amenorrhoea, nocturia and polydipsia secondary to anterior and posterior pituitary hormone deficiencies. Menstrual irregularities are common in women with pituitary abscesses likely because the gonadotrophs are more easily disrupted than other hormone-secreting cells of the anterior pituitary (5). The presence of diabetes insipidus can help discriminate the cause of a suprasellar mass and pituitary hormone deficiencies. It is uncommon for pituitary adenomas to present with diabetes insipidus (2). However, other suprasellar masses, such as pituitary abscesses, often present with diabetes insipidus (6).

MRI is the preferred imaging modality for the pathology of the pituitary. However, MRI has limitations in delineating pituitary abscesses from other pathology of the suprasellar region. Pituitary abscesses that occur because of a predisposing anatomic abnormality, such as a craniopharyngioma, may have an appearance that is similar to the predisposing anatomic abnormality (7). This patient presented with a peripherally enhancing, cystic, suprasellar mass, which is an MRI appearance that has been seen in cases of pituitary abscess but is not specific for pituitary abscess (7). The patient also did not have the posterior pituitary bright spot on MRI, which is associated with diabetes insipidus (8). As diabetes insipidus does not frequently occur because of pituitary adenomas, the absence of the posterior pituitary bright spot on MRI should prompt clinicians to entertain other diagnoses (7).

Pituitary abscess is often not diagnosed until the time of surgery because it presents much like other suprasellar mass lesions and patients often do not have signs of infection. In one series, the diagnosis of pituitary abscess was made preoperatively in only $20.8 \%$ of patients (1). As seen in our patient, MRI is not always able to discriminate between pituitary abscess and other suprasellar lesions. The diagnosis of pituitary abscess requires a high degree of clinical suspicion, as only a third of patients with pituitary abscess present with signs of infection such as fever or leucocytosis (1).

The patient had surgical cultures that showed two species of alpha hemolytic Streptococcus, Staphylococcus capitis and Prevotella melaninogenica. Many different organisms have been seen in surgical cultures from pituitary abscess; however, Gram-positive organisms such as Streptococcus and Staphylococcus are the most common (1). Anaerobic organisms such as P. melaninogenica have been implicated in at least one other case of pituitary abscess but are much less common (9). P. melaninogenica is considered normal oral flora that could be suggestive of an underlying dental infection. However, dental examination and dental radiographs revealed no sign of infection.

Transsphenoidal resection and antibiotic therapy is the mainstay of treatment for pituitary abscess. Transsphenoidal resection is preferred over craniotomy, as craniotomy is more invasive and could result in spillage of infectious material into the CSF (10). The patient received 6 weeks or oral metronidazole and intravenous vancomycin based on the findings from her surgical cultures. Antibiotic therapy is typically tailored to surgical cultures. The majority of patients with positive surgical Gram stain or cultures receive antibiotic therapy for 4-6 weeks (1).

Appropriate and timely treatment of pituitary abscess typically results in low mortality. In one series, only $8.3 \%$ of patients died from complications of pituitary abscess (1). Compressive symptoms such as headache and visual changes also respond favourably to treatment. Over $90 \%$ of patients noted improvement in headaches and $75 \%$ of patients had improvement in visual changes 
in one series (1). The patient presented in the case had resolution of her headaches and bitemporal hemianopsia after treatment. As seen in this patient, pituitary hormone deficiencies often persist following treatment of pituitary abscess. About $62.5 \%$ of patients with pituitary abscess presenting with new pituitary hormone deficiencies required long-term hormone replacement (1).

\section{Declaration of interest}

The authors declare that there is no conflict of interest that could be perceived as prejudicing the impartiality of the research reported.

\section{Funding}

This research did not receive any specific grant from any funding agency in the public, commercial or not-for-profit sector

\section{Patient consent}

A written informed consent has been obtained from the patient for publication of the submitted article and accompanying images.

\section{Author contributions and acknowledgements}

D Adams is an advanced trainee in Endocrinology who looked after the patient. He also wrote the initial draft for this manuscript. P Kern is a consultant endocrinologist, who was the physician responsible for the patient's care at different times. He also reviewed and revised this manuscript.

\section{References}

1 Vates GE, Berger MS \& Wilson CB 2001 Diagnosis and management of pituitary abscess: a review of twenty-four cases. Journal of Neurosurgury 95 233-241. (doi:10.3171/jns.2001.95.2.0233)

2 Askenasy HM, Israeli J, Karny H \& Dujovny M 1971 Intrasellar abscess simulating pituitary adenoma. Neurochirurgia 14 34-37. (doi:10.1055/s-0028-1090553)

3 Dalan R \& Leow MK 2008 Pituitary abscess: our experience with a case and a review of the literature. Pituitary 11 299-306. (doi:10.1007/ s11102-007-0057-3)

4 Dutta P, Bhansali A, Singh P, Kotwal N, Pathak A \& Kumar Y 2006 Pituitary abscess: report of four cases and review of literature. Pituitary 9 267-273. (doi:10.1007/s11102-006-8327-z)

5 Littley MD, Shalet SM, Beardwell CG, Ahmed SR, Applegate G \& Sutton ML 1989 Hypopituitarism following external radiotherapy for pituitary tumours in adults. QJM 70 145-160.

6 Ford J, Torres LF, Cox T \& Hayward R 1986 Recurrent sterile meningitis caused by a pituitary abscess. Postgraduate Medical Journal 62 929-931. (doi:10.1136/pgmj.62.732.929)

7 Wolansky LJ, Gallagher JD, Heary RF, Malantic GP, Dasmahapatra A, Shaderowfsky PD \& Budhwani N 1997 MRI of pituitary abscess: two cases and review of the literature. Neuroradiology 39 499-503. (doi:10.1007/s002340050453)

8 Shin JH, Lee HK, Choi CG, Suh DC, Kim CJ, Hong SK \& Na DG 2001 MR imaging of central diabetes insipidus: a pictorial essay. Korean Journal of Radiology 2 222-230. (doi:10.3348/kjr.2001.2.4.222)

9 Beatty N, Medina-Garcia L, Al Mohajer M \& Zangeneh TT 2016 Polymicrobial pituitary abscess predominately involving Escherichia coli in the setting of an apoplectic pituitary prolactinoma. Case Reports in Infectious Diseases 2016 1-5. (doi:10.1155/2016/4743212)

10 Zhang X, Sun J, Shen M, Shou X, Qiu H, Qiao N, Zhang N, Li S, Wang Y \& Zhao Y 2012 Diagnosis and minimally invasive surgery for the pituitary abscess: a review of twenty nine cases. Clinical Neurology and Neurosurgery 114 957-961. (doi:10.1016/j. clineuro.2012.02.020)

Received in final form 1 July 2016

Accepted 18 July 2016 